\section{Beneficio de la quimioterapia adyuvante en pacientes con cáncer de colon: cohorte retrospectiva de un hospital clínico universitario}

\author{
SEBASTIÁN MONDACA ${ }^{1}$, CONSTANZA VILLALÓN $^{2}$, JOSÉ LUIS LEAL ${ }^{1}$, \\ ÁLVARO ZÚÑIGA ${ }^{2}$, FELIPE BELLOLIO $^{2}$, OSLANDO PADILLA $^{3, a}$, \\ SILVIA PALMA $^{1, \mathrm{~b}}$, MARCELO GARRIDO ${ }^{1}$, BRUNO NERVI $^{1}$
}

\section{Benefit of adjuvant 5-fluorouracil based chemotherapy for colon cancer: a retrospective cohort study}

Background: Multiple clinical trials have demonstrated the benefits of adjuvant 5-fluorouracil-based chemotherapy for patients with resectable colon cancer (CC), especially in stage III. Aim: To describe the clinical characteristics of a cohort of CC patients treated at a single university hospital in Chile since 2002, and to investigate if chemotherapy had an effect on survival rates. Material and Methods: Review of a tumor registry of the hospital. Medical records of patients with CC treated between 2002 and 2012 were reviewed. Death certificates from the National Identification Service were used to determine mortality. Overall survival was described using the Kaplan-Meier method. A multivariate Cox proportional hazard regression model was also used. Results: A total of 370 patients were treated during the study period (202 in stage II and 168 in stage III). Adjuvant chemotherapy was administered to 22 and $70 \%$ of patients in stage II and III respectively. The median follow-up period was 4.6 years. The 5-year survival rate for stage II patients was 79\% and there was no benefit observed with adjuvant chemotherapy. For stage III patients, the 5-year survival rate was $81 \%$ for patients who received adjuvant chemotherapy, compared to $56 \%$ for those who did not receive chemotherapy (hazard ratio (HR): 0.29; 95\% confidence interval (CI): 0.15-0.56). The benefit of chemotherapy was found to persist after adjustment for other prognostic variables (HR: 0.47; 95\% CI: 0.23-0.94).Conclusions: Patients with colon cancer in stage III who received adjuvant chemotherapy had a better overall survival.

(Rev Med Chile 2016; 144: 145-151)

Key words: Antineoplastic combined chemotherapy protocols; Chemotherapy, adjuvant; Colonic neoplasms; Fluorouracil.
'Departamento de Hematología y Oncología, Pontificia Universidad Católica de Chile, Santiago, Chile. 2Departamento de Cirugía Digestiva, Pontificia Universidad Católica de Chile, Santiago, Chile. ${ }^{3}$ Departamento de Salud Pública, Pontificia Universidad Católica de Chile, Santiago, Chile.

aEstadístico.

${ }^{\text {b}}$ Enfermera Universitaria.

Recibido el 5 de junio de 2015, aceptado el 16 de noviembre de 2015.

Correspondencia a:
Bruno Nervi
Departamento de Hematología
y Oncología. Centro de Cáncer.
Pontificia Universidad Católica
de Chile.
Diagonal Paraguay 319, Santiago,
Chile.
Código postal 8330032
Teléfonos: 2-3546919
Fax: 2-2472327
bnervi@med.puc.cl

$\mathrm{E}$ 1 cáncer de colon (CC) es la tercera neoplasia en frecuencia y la cuarta en mortalidad a nivel mundial ${ }^{1}$. A diferencia de lo que ocurre en países desarrollados, donde su mortalidad va en descenso ${ }^{2,3}$, en Chile habría una tendencia al alza en su mortalidad de 3,7 en el año 1990 a
6,3/100.000 en el año 2004 . Este hecho puede ser explicado por múltiples hipótesis, destacando cambios en el estilo de vida de la población, falta de acceso a programas de tamizaje en población asintomática y diferencias en las oportunidades de tratamiento de esta enfermedad. 
La quimioterapia (QT) adyuvante ha sido extensamente estudiada y desde la década 199099 se demostró un beneficio en sobrevida global cercano a $10 \%$ con quimioterapia basada en 5-fluorouracilo (5 FU) modulado con leucovorina (LV) en pacientes con CC en etapa III (con metástasis ganglionares regionales) $)^{5-7}$. Se han estudiado múltiples formas de administración de estas drogas, siendo los esquemas infusionales los más utilizados en los últimos años, tanto en la adyuvancia como en el escenario metastásico ${ }^{8}$. Posteriormente, los estudios MOSAIC ${ }^{9}$ y NSABP $\mathrm{C}-07^{10}$ mostraron un mayor beneficio al agregar la droga oxaliplatino (esquema FOLFOX) logrando una mejora en sobrevida global de $4 \%$ a 5 años.

Estos resultados no fueron categóricos en pacientes en etapa II ( $\sin$ metástasis ganglionares regionales $)^{11-13}$, por lo que para estos pacientes las guías internacionales sólo recomiendan tratamiento con QT adyuvante en caso de factores pronósticos adversos ${ }^{14,15}$. La capecitabina es una prodroga del $5 \mathrm{FU}$ de administración por vía oral que ha mostrado resultados equivalentes en adyuvancia $^{16,17}$, por lo que se considera una alternativa de tratamiento tanto como monoterapia como en combinación con oxaliplatino.

El objetivo de este estudio es describir a la población de pacientes que recibieron quimioterapia adyuvante por cáncer de colon en el Hospital de la Universidad Católica de Chile, y observar su influencia en la sobrevida libre de enfermedad y sobrevida global.

\section{Material y Método}

En este estudio se incluyeron todos los pacientes con CC tipo adenocarcinoma etapas II y III sometidos a resección quirúrgica con intención curativa en el Hospital Clínico de la Universidad Católica de Chile entre enero de 2002 y junio de 2012. Se excluyeron los pacientes con cáncer de recto, definidos como tumor hasta $15 \mathrm{~cm}$ sobre margen anal y los que realizaron su tratamiento adyuvante en otro centro. Los datos fueron recolectados de la ficha clínica de cada paciente y del registro de tumores de la institución. La información relativa a mortalidad se extrajo de la base de datos del Servicio de Registro Civil de Chile ${ }^{18}$. Para la estadificación se utilizó la clasificación de la AJCC TNM $7^{\text {a }}$ edición ${ }^{19}$. Los pacientes con depósitos tumorales en el tejido adiposo pericolónico se consideraron como etapa III sin sumarse éstos al número total de ganglios. A todos los pacientes se les realizó una tomografía axial computada de tórax, abdomen y pelvis para descartar enfermedad metastásica al momento del diagnóstico. Se realizó seguimiento clínico, con antígeno carcinoembrionario (CEA) y mediante imágenes, hasta completar 5 años del término del tratamiento.

Los pacientes recibieron quimioterapia adyuvante según la recomendación de su oncólogo tratante. Para la decisión en pacientes en etapa III se consideraron la edad, las comorbilidades del paciente y la existencia de complicaciones postoperatorias. En el caso de los pacientes en etapa II se tomaron en cuenta además la presencia de factores de mal pronóstico como: tumor T4, perforación intestinal, tumor obstructivo, mala diferenciación, menos de 12 ganglios a la patología, permeaciones linfovasculares y CEA elevado antes de la cirugía. Durante el período de estudio fueron variando los esquemas de quimioterapia utilizados según a la evidencia disponible, destacando las combinaciones de $5 \mathrm{FU} / \mathrm{LV}$ (esquema $\mathrm{Mayo}^{20} \mathrm{o}$ infusional) y FOLFOX $^{21}$ (Tabla 1).

Tabla 1. Esquemas de quimioterapia en cáncer de colon

\begin{tabular}{|lll|}
\hline Esquema & Drogas & Administración \\
\hline $5 \mathrm{FU} / \mathrm{LV}$ & $5 \mathrm{FU} 425 \mathrm{mg} / \mathrm{m}^{2}$, & Cada 1 mes por 6 ciclos \\
Mayo & LV $20 \mathrm{mg} / \mathrm{m}^{2}$ días $1-5$ & \\
5 FU/LV Infusional & $5 \mathrm{FU} 400 \mathrm{mg} / \mathrm{m}^{2}$ día 1 y 2, & Cada 2 semanas por 12 ciclos \\
& $5 \mathrm{FU} 600 \mathrm{mg} / \mathrm{m}^{2}$ infusión día 1 y 2, & \\
& LV $200 \mathrm{mg} / \mathrm{m}^{2}$ día 1 y 2 & \\
FOLFOX & 5 FU $400 \mathrm{mg} / \mathrm{m}^{2}$ día 1 y $2,5 \mathrm{FU} 600 \mathrm{mg} / \mathrm{m}^{2}$ infusión día 1 y 2, & Cada 2 semanas por 12 ciclos \\
& LV $200 \mathrm{mg} / \mathrm{m}^{2}$ día 1 y 2, oxaliplatino $85 \mathrm{mg} / \mathrm{m}^{2}$ día 1 & \\
\hline
\end{tabular}

5 FU: 5 Fluorouracilo; LV: Leucovorina. 
Las características de la población se expresaron como promedios para las variables continuas y como porcentajes para las variables categóricas. El análisis estadístico se realizó mediante t-Student para las variables continuas, $\chi^{2}$ para las variables categóricas y curvas de Kaplan-Meier con test de log-rank para el análisis de sobrevida. Se realizó un análisis univariado y posteriormente multivariado mediante regresión de Cox para detectar variables pronósticas independientes. El análisis de datos se realizó mediante el programa SPSS versión 21. Este estudio fue aprobado por el comité de ética institucional.

\section{Resultados}

\section{Características de los pacientes}

En este estudio se incluyeron 370 pacientes tratados entre enero de 2002 y junio de 2012 . Se excluyó a 10 pacientes por tener un registro incompleto. El 49\% de los pacientes correspondieron a hombres y la edad promedio fue 67,5 años, con un rango de 20 a 94 años. La localización anatómica del tumor fue en el colon izquierdo en $42 \%$ y en el colon derecho en $40 \%$ de los casos. La histología correspondió en 93,5\% a adenocarcinoma tubular, mientras que la variante mucinosa fue la segunda en frecuencia con 5,9\% del total (Tabla 2).

Fueron diagnosticados en etapa II y en etapa III, 202 y 168 pacientes respectivamente. De los pacientes en etapa II, 58,9\% presentaba algún factor de mal pronóstico, proporción que fue mayor en pacientes que recibieron quimioterapia en comparación a los que no recibieron $(86,6 \%$ versus $50,5 \%$; $p<0,01$ ). Los factores de riesgo más prevalentes en este grupo fueron las permeaciones linfovasculares, con 22,7\%, y los tumores T4 o perforados, con 13,3\% (Tabla 3).

En los pacientes en etapa III, el número promedio de ganglios con metástasis fue de 4,1. En este grupo 59\% tuvieron 1 a 3 ganglios comprometidos por tumor, $27 \% 4$ a 6 y $13 \%$ igual o más de 7 .

\section{Tratamiento y sobrevida}

La mediana de seguimiento en este estudio fue de 4,6 años. La edad promedio de los pacientes que recibieron QT fue de 59 años, mientras la de los que no recibieron fue de 74 años $(\mathrm{p}<0,01)$. Se administró QT a 45 de 202 (22,2\%) pacientes en etapa II y a 117 de $168(69,6 \%)$ en etapa III. En
Tabla 2. Características de los pacientes

$\left.\begin{array}{|lcc|}\hline & \begin{array}{c}\text { Etapa II } \\ \mathbf{n = 2 0 2}\end{array} & \begin{array}{c}\text { Etapa III } \\ \mathbf{n = 1 6 8}\end{array} \\ \text { Edad } & 69 & 65,8 \\ \text { Sexo } & & \\ \text { Masculino } & 49 & 50,5 \\ \text { Femenino } & 50,9 & 49,4\end{array}\right)$

Adenoca: Adenocarcinoma.

Tabla 3. Factores de riesgo en etapa II

\begin{tabular}{|lc|}
\hline & $\begin{array}{c}\text { Etapa II } \\
\mathbf{n}=\mathbf{2 0 2}\end{array}$ \\
\hline T4/perforado & 13,3 \\
\hline Obstrucción intestinal & 7,9 \\
\hline Menos 12 ganglios & 3,4 \\
\hline PLV $(+)$ & 22,7 \\
\hline Mala diferenciación & 6,4 \\
\hline CEA elevado & 4,9 \\
\hline Total & $58,9 \%$ \\
\hline
\end{tabular}

CEA: Antígeno carcinoembrionario. PLV: Permeación linfovascular.

el primer grupo, el esquema más usado fue $5 \mathrm{FU} /$ $\mathrm{LV}$, en $76,7 \%$ de los casos, y en el segundo grupo fue FOLFOX, en $60,1 \%$ (Tabla 4).

La sobrevida a 5 años fue de $79 \%$ en el grupo en etapa II y de $74 \%$ en el grupo en etapa III 
Tabla 4. Esquemas de quimioterapia utilizados por etapa

\begin{tabular}{|lcc|}
\hline & $\begin{array}{c}\text { Etapa II } \\
\mathbf{n = 4 5}\end{array}$ & $\begin{array}{c}\text { Etapa III } \\
\mathbf{n = 1 1 7}\end{array}$ \\
\hline FOLFOX & 18,6 & 60,1 \\
5 FU/LV & 76,7 & 38 \\
\hline Capecitabina & 4,6 & 1,7 \\
\hline
\end{tabular}

5 FU: 5 Fluorouracilo; LV: Leucovorina.

(Figura 1). Los pacientes en etapa II con factores de riesgo tuvieron una tendencia a peor sobrevida a 5 años respecto a los que no los presentaban $(73 \%$ versus $87 \%$ ), si bien esto no fue estadísticamente significativo, HR 1,29 (IC 95\% 0,69-2,41) (Figu- ra 2). Al considerar el efecto de la quimioterapia en este estadio observamos que la sobrevida a 5 años fue de $80 \%$ en el grupo tratado y $79 \%$ en el grupo no tratado. La sobrevida libre de enfermedad a 5 años fue 84\% en ambos grupos HR 1,04 (IC 95\% $0,44-2,52)$.

En los pacientes en estadio III se observó una relación entre el compromiso ganglionar y un peor pronóstico, con una sobrevida a 5 años en los pacientes con $<20 \%, 20-40 \%$ y $>40 \%$ ganglios comprometidos por tumor, de $84 \%$, $58 \%$ y $40 \%$ respectivamente $(\mathrm{p}<0,05)$.

Los pacientes que recibieron quimioterapia en este grupo tuvieron una sobrevida a 5 años de $81 \%$ comparado con $56 \%$ en los que no la recibieron, HR 0,29 (IC 95\% 0,15-0,56) (Figura 3). La sobrevida libre de enfermedad a 3 años fue mejor en el

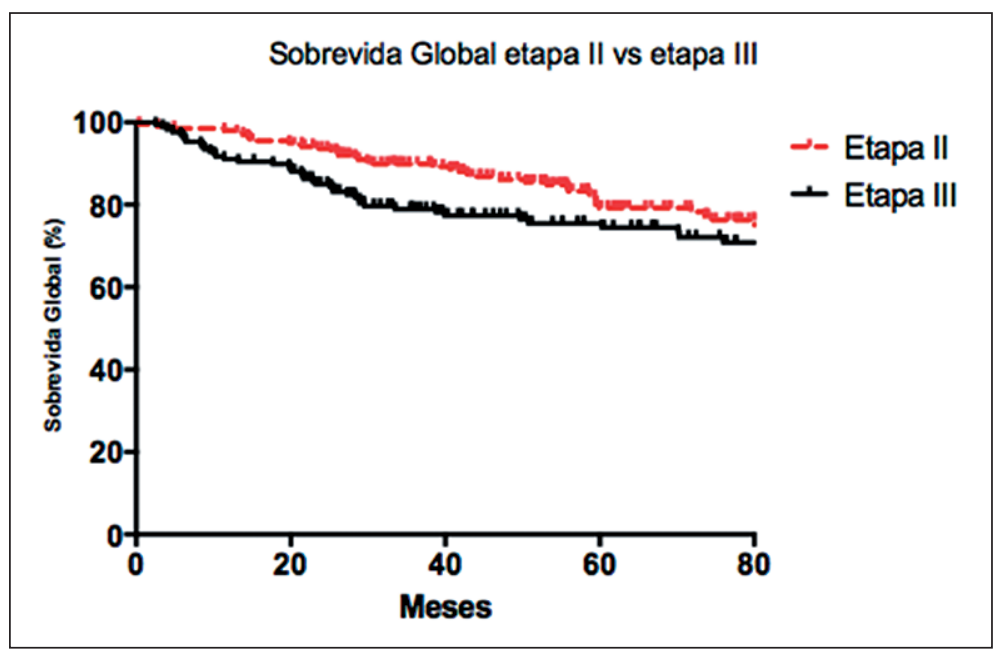

Figura 1. Curva de sobrevida global por etapa.

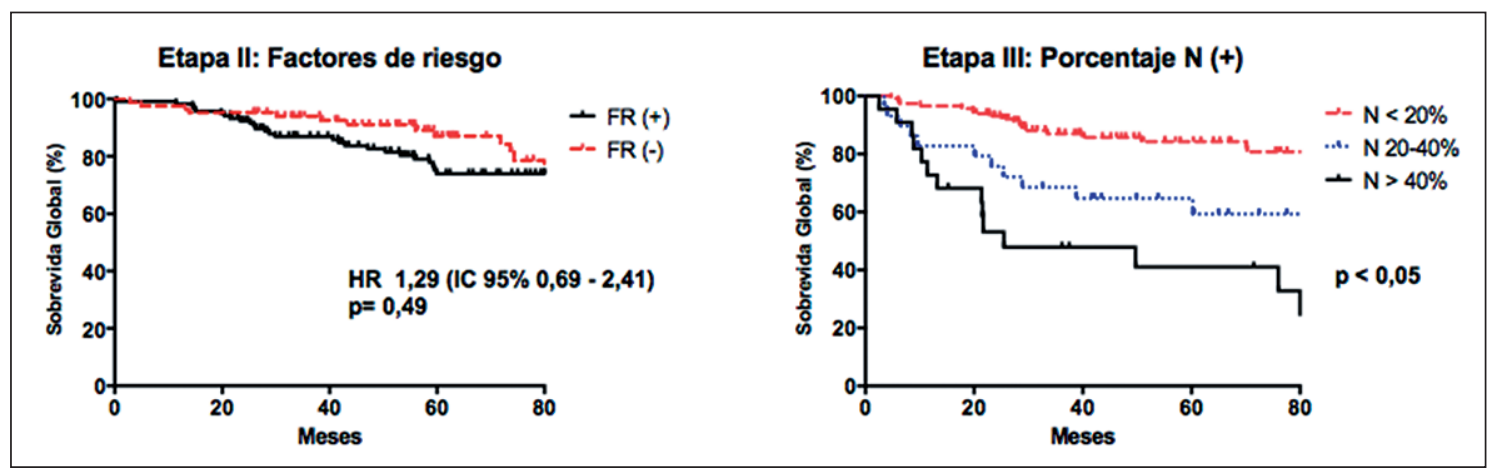

Figura 2. Curvas de sobrevida según factores de riesgo y porcentaje de ganglios positivos. 


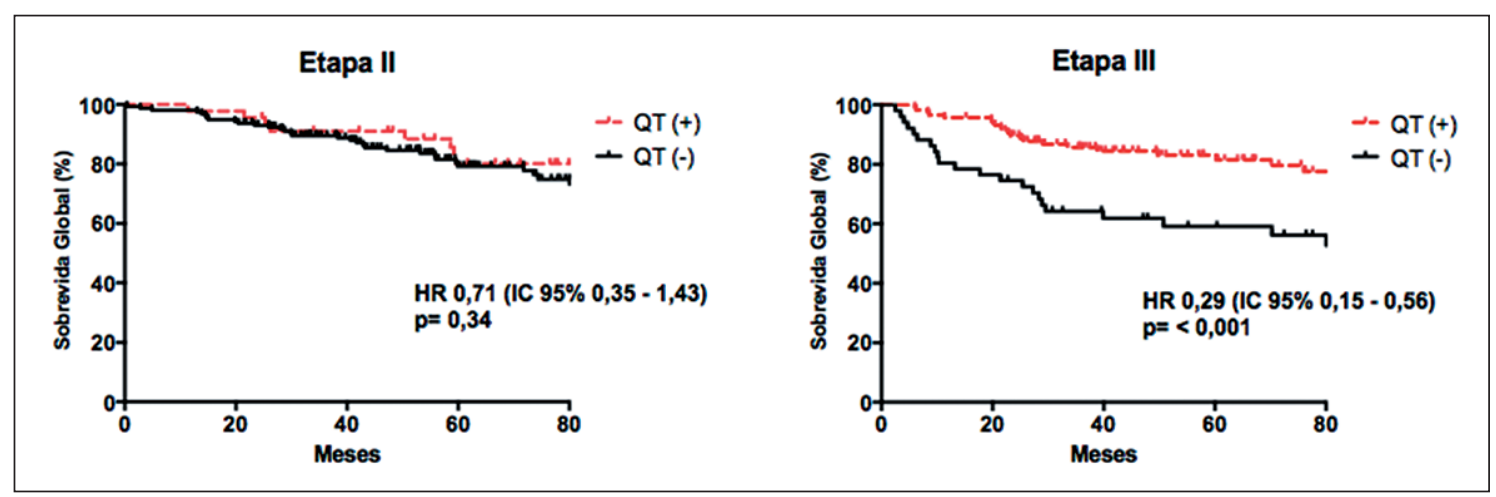

Figura 3. Curvas de sobrevida global según la administración de quimioterapia.

Tabla 5. Análisis multivariado de factores relacionados a sobrevida

\begin{tabular}{|lccl|}
\hline & HR & IC $\mathbf{9 5 \%}$ & p-value \\
Etapa II & & & \\
$\quad$ Edad & 1,04 & $1,009-1,075$ & 0,012 \\
FR $(+)$ & 1,12 & $0,46-2,84$ & 0,8 \\
QT adyuvante & 1,21 & $0,62-2,35$ & 0,56 \\
Etapa III & & & \\
Edad & 1,02 & $0,99-1,05$ & 0,06 \\
> 6 ganglios (+) & 2,51 & $1,13-5,5$ & 0,023 \\
PLV $(+)$ & 2,3 & $1,12-4,7$ & 0,023 \\
QT adyuvante & 0,47 & $0,23-0,94$ & 0,03 \\
\hline
\end{tabular}

FR: Factor de riesgo. QT: quimioterapia. PLV: Permeación Linfovascular.

grupo tratado respecto al no tratado, $78 \%$ versus $73 \%$, pero a 5 años no hubo diferencias con $67 \%$ versus $66 \%$, HR 0,87 (IC 95\% 0,47-1,64). No se encontraron diferencias significativas entre los distintos esquemas de quimioterapia utilizados.

Tanto en el análisis univariado como en el multivariado se encontró una influencia significativa de la QT en el riesgo de mortalidad para los pacientes en etapa III, HR 0,47 (IC 95\% 0,23$0,94)$. Por el contrario, en los pacientes en etapa II la quimioterapia no se asoció a beneficio, HR 1,21 (IC 95\% 0,62-2,35) (Tabla 5).

\section{Discusión}

En nuestro conocimiento, la presente cohorte de pacientes con CC tratados con QT adyuvante corresponde a la más grande publicada en Chile hasta la fecha, y es la primera que demuestra beneficio en sobrevida.

En este estudio, la recomendación de QT dependió del médico tratante. El porcentaje de pacientes en etapa II y III que recibió QT en esta serie fue de 22 y $70 \%$ respectivamente. Esto es similar a la frecuencia reportada por un estudio americano utilizando la base de datos del SEER (Surveillance, Epidemiology, and End Results) que incluyó a más de 23.000 etapa II y 17.000 pacientes en etapa $\mathrm{III}^{22}$. Los datos obtenidos en nuestro estudio reproducen la sobrevida alcanzada en estudios aleatorizados internacionales en esta patología $a^{5,9,10}$.

También cabe destacar que la presente cohorte incluye pacientes con riesgo significativo de recaída. Por ejemplo, de los pacientes en etapa II, más de $50 \%$ contaba con uno o más factores de mal pronóstico, mientras que en los etapa III, 40\% tenía 4 o más ganglios comprometidos por tumor. Una limitación del presente estudio es que no se realizó análisis de inestabilidad microsatelital. Este fenómeno es un signo biológico de falla en la reparación del ADN que está presente en 15 a 20\% de los pacientes con CC localizado y se asociaría a ausencia de beneficio de QT basada en $5 \mathrm{FU}^{23,24}$ $\mathrm{y}$ a un menor riesgo de recidiva ${ }^{25}$.

Dentro de los resultados destaca una mejor sobrevida en los pacientes etapa III tratados con quimioterapia en comparación a los no tratados. Si bien, al no haber un grupo de control de similares características existe un sesgo de selección significativo, esta diferencia se mantiene al realizar el análisis multivariado. La diferencia a favor 
de la QT nos parece relevante del punto de vista clínico y difícil de explicar solamente por el sesgo inherente a un estudio observacional.

No se observaron diferencias en sobrevida entre pacientes tratados con QT con o sin oxaliplatino, lo que se contradice con los estudios de adyuvancia mencionados ${ }^{9,10}$. Esto se podría explicar por el relativo bajo poder del estudio para demostrar diferencias en este desenlace.

Respecto a los pacientes en etapa II, se observa una sobrevida a 5 años cercana a $80 \%$, la cual es similar a la reportada en estudios de adyuvancia como el estudio QUASAR ${ }^{26} \mathrm{o}$ la base de registro del SEER ${ }^{27}$. Destaca que los pacientes tratados con QT tuvieron una sobrevida similar a los no tratados, pese a que los primeros tenían una mayor prevalencia de factores de mal pronóstico. Estos resultados podrían sugerir que la QT sería capaz de compensar el mal pronóstico determinado por la existencia de estos factores. Otros trabajos ya han sugerido este beneficio, como la base de estudios aleatorios ACCENT en la que se determinó un beneficio de $5 \%$ en sobrevida a 8 años $^{28}$; esto contrasta con otros análisis cooperativos que sólo han mostrado beneficio en sobrevida libre de enfermedad ${ }^{29}$.

Un estudio chileno de Bannura et al. ${ }^{30}$ también exploró en forma observacional el beneficio de la QT adyuvante en cáncer de colon, mostrando una tendencia al beneficio con el esquema de $5 \mathrm{FU} / \mathrm{LV}$ en bolo, pero la diferencia no fue estadísticamente significativa. Si bien ambas iniciativas realizadas en esta población aportan en el estudio de esta enfermedad, el número de pacientes que consideran es pequeño al compararlo con otras bases de registro. Por ejemplo, la base del SEER cubre los registros de $26 \%$ de la población norteamericana, lo que ha permitido el desarrollo de estudios observacionales con un poder adecuado y buena calidad metodológica ${ }^{31}$.

Dentro de las fortalezas de nuestro estudio destaca que se realizó en un solo centro universitario y que cuenta con un seguimiento prolongado. Además, a partir del año 2007 un porcentaje muy relevante de los pacientes fueron tratados con la QT estándar hasta la fecha (FOLFOX).

En conclusión, nuestro estudio apoya el beneficio de la QT adyuvante en pacientes con CC en etapa III, y sugiere un beneficio para pacientes en etapa II con factores de riesgo. En este último grupo es necesario desarrollar mejores marcadores predictivos para así identificar a los pacientes que realmente se benefician de este tratamiento.

\section{Referencias}

1. Ferlay J, Shin H, Bray F, Forman D, Mathers C, Parkin D. GLOBOCAN 2008-cancer incidence and mortality worldwide: IARC Cancer Base $\mathrm{N}^{\circ} 10$. Lyon: International Agency for Research on Cancer http://globocan.iarc. fr2010 [citado el 4 de mayo de 2013].

2. Center MM, Jemal A, Smith RA, Ward E. Worldwide variations in colorectal cancer. CA Cancer J Clin 2009; 59 (6): 366-78.

3. Siegel R, Naishadham D, Jemal A. Cancer statistics, 2013. CA Cancer J Clin 2013; 63 (1): 11-30.

4. Donoso A, Villarroel L, Pinedo G. [Increase in colon cancer mortality rates in Chile, during the period 19902003]. Rev Med Chile 2006; 134 (2): 152-8.

5. Efficacy of adjuvant fluorouracil and folinic acid in colon cancer. International Multicentre Pooled Analysis of Colon Cancer Trials (IMPACT) investigators. Lancet 1995; 345 (8955): 939-44.

6. Macdonald JS. Adjuvant therapy of colon cancer. CA Cancer J Clin 1999; 49 (4): 202-19.

7. Gill S, Loprinzi CL, Sargent DJ, Thomé SD, Alberts SR, Haller DG, et al. Pooled analysis of fluorouracil-based adjuvant therapy for stage II and III colon cancer: who benefits and by how much? J Clin Oncol 2004; 22 (10): 1797-806.

8. de Gramont A, Bosset JF, Milan C, Rougier P, Bouché $\mathrm{O}$, Etienne PL, et al. Randomized trial comparing monthly low-dose leucovorin and fluorouracil bolus with bimonthly high-dose leucovorin and fluorouracil bolus plus continuous infusion for advanced colorectal cancer: a French intergroup study. J Clin Oncol 1997; 15 (2): 808-15.

9. André T, Boni C, Navarro M, Tabernero J, Hickish $\mathrm{T}$, Topham C, et al. Improved overall survival with oxaliplatin, fluorouracil, and leucovorin as adjuvant treatment in stage II or III colon cancer in the MOSAIC trial. J Clin Oncol 2009; 27 (19): 3109-16.

10. Yothers G, O’Connell MJ, Allegra CJ, Kuebler JP, Colangelo LH, Petrelli NJ, et al. Oxaliplatin as adjuvant therapy for colon cancer: updated results of NSABP C-07 trial, including survival and subset analyses. J Clin Oncol 2011; 29 (28): 3768-74.

11. Efficacy of adjuvant fluorouracil and folinic acid in B2 colon cancer. International Multicentre Pooled Analysis of B2 Colon Cancer Trials (IMPACT B2) Investigators. J Clin Oncol 1999; 17 (5): 1356-63. 
12. Buyse M, Piedbois P. Should Dukes' B patients receive adjuvant therapy? A statistical perspective. Semin Oncol 2001; 28 (1 Suppl 1): 20-4.

13. Figueredo A, Coombes ME, Mukherjee S. Adjuvant therapy for completely resected stage II colon cancer. Cochrane Database Syst Rev 2008 (3): CD005390.

14. NCCN Clinical Practice Guidelines in Oncology: Colon Cancer. Version 1 http://www.nccn.org/professionals/ physician_gls/f_guidelines.asp2014 [cited 2014 Mayo 26].

15. Labianca R, Nordlinger B, Beretta GD, Mosconi S, Mandalà $\mathrm{M}$, Cervantes $\mathrm{A}$, et al. Early colon cancer: ESMO Clinical Practice Guidelines for diagnosis, treatment and follow-up. Ann Oncol 2013; 24 Suppl 6: vi64-72.

16. Twelves C, Wong A, Nowacki MP, Abt M, Burris H, Carrato A, et al. Capecitabine as adjuvant treatment for stage III colon cancer. N Engl J Med 2005; 352 (26): 2696-704.

17. Haller DG, Tabernero J, Maroun J, de Braud F, Price T, Van Cutsem E, et al. Capecitabine plus oxaliplatin compared with fluorouracil and folinic acid as adjuvant therapy for stage III colon cancer. J Clin Oncol 2011; 29 (11): 1465-71.

18. Registro Civil e identificación de Chile 2013. https:// http://www.registrocivil.cl/OficinaInternet/servlet/HomeVenta on October 10, 2013 [cited 2013 October 10].

19. Sobin L, Gospodarowicz M, Wittekind C. TNM classification of malignant tumours. New York: Wiley-Blackwell 2009.

20. O'Connell MJ, Mailliard JA, Kahn MJ, Macdonald JS, Haller DG, Mayer RJ, et al. Controlled trial of fluorouracil and low-dose leucovorin given for 6 months as postoperative adjuvant therapy for colon cancer. J Clin Oncol 1997; 15 (1): 246-50.

21. de Gramont A, Figer A, Seymour M, Homerin M, Hmissi A, Cassidy J, et al. Leucovorin and fluorouracil with or without oxaliplatin as first-line treatment in advanced colorectal cancer. J Clin Oncol 2000; 18 (16): 2938-47.

22. O'Connor ES, Greenblatt DY, Lo Conte NK, Gangnon RE, Liou JI, Heise CP, et al. Adjuvant chemotherapy for stage II colon cancer with poor prognostic features. J
Clin Oncol 2011; 29 (25): 3381-8.

23. Ribic CM, Sargent DJ, Moore MJ, Thibodeau SN, French AJ, Goldberg RM, et al. Tumor microsatellite-instability status as a predictor of benefit from fluorouracil-based adjuvant chemotherapy for colon cancer. N Engl J Med 2003; 349 (3): 247-57.

24. Des Guetz G, Schischmanoff O, Nicolas P, Perret GY, Morere JF, Uzzan B. Does microsatellite instability predict the efficacy of adjuvant chemotherapy in colorectal cancer? A systematic review with meta-analysis. Eur J Cancer 2009; 45 (10): 1890-6.

25. Gryfe R, Kim H, Hsieh ET, Aronson MD, Holowaty EJ, Bull SB, et al. Tumor microsatellite instability and clinical outcome in young patients with colorectal cancer. $\mathrm{N}$ Engl J Med 2000; 342 (2): 69-77.

26. Gray R, Barnwell J, McConkey C, Hills RK, Williams NS, Kerr DJ, et al. Adjuvant chemotherapy versus observation in patients with colorectal cancer: a randomised study. Lancet 2007; 370 (9604): 2020-9.

27. O’Connell JB, Maggard MA, Ko CY. Colon cancer survival rates with the new American Joint Committee on Cancer sixth edition staging. J Natl Cancer Inst 2004; 96 (19): 1420-5.

28. Sargent D, Sobrero A, Grothey A, O'Connell MJ, Buyse M, Andre T, et al. Evidence for cure by adjuvant therapy in colon cancer: observations based on individual patient data from 20,898 patients on 18 randomized trials. J Clin Oncol 2009; 27 (6): 872-7.

29. Figueredo A, Charette ML, Maroun J, Brouwers MC, Zuraw L. Adjuvant therapy for stage II colon cancer: a systematic review from the Cancer Care Ontario Program in evidence-based care's gastrointestinal cancer disease site group. J Clin Oncol 2004; 22 (16): 3395-407.

30. Bannura G, Castillo C, Cumsille M, Barrera A. Resultados de la quimioterapia adyuvante en cáncer de colon. Rev Chilena de Cirugía p. 403-9.

31. Sanoff HK, Carpenter WR, Stürmer T, Goldberg RM, Martin CF, Fine JP, et al. Effect of adjuvant chemotherapy on survival of patients with stage III colon cancer diagnosed after age 75 years. J Clin Oncol 2012; 30 (21): 2624-34. 